\title{
Energy loss by fast-travelling charged particles traversing two-dimensional materials
}

\author{
Jaime E. Santos ${ }^{1, *}$, Mikhail Vasilevskiy ${ }^{1,2}$, Nuno M. R. Peres ${ }^{1,2}$, and Antti-Pekka Jauho ${ }^{3}$ \\ ${ }^{1}$ Centro de Física e Departamento de Física, Universidade do Minho, 4710-057 Braga, Portugal \\ ${ }^{2}$ International Iberian Nanotechnology Laboratory, Avenida Mestre José Veiga s/n, 4715-330 Braga, \\ Portugal \\ ${ }^{3}$ Center for Nanostructured Graphene (CNG), Department of Physics DTU, building 345C, 2800 Kgs. \\ Lyngby, Denmark
}

\begin{abstract}
We consider the problem of the radiation losses by fast-traveling particles traversing two-dimensional (2d) materials or thin films. After reviewing the screening of electromagnetic fields by two dimensional conducting materials, we obtain the energy loss by a fast particle traversing such a material or film. In particular, we discuss the pattern of radiation emitted by monolayer graphene treated within a hydrodynamic approximation. These results are compared with recent published results using similar approximations and, having in mind a potential application to particle detection, we briefly discuss how one can improve on the signals obtained by using other two-dimensional materials.
\end{abstract}

\section{Introduction}

The problem of the interaction of the electromagnetic field with a $2 \mathrm{~d}$ conductor has a long and distinguished history, going back to the 1950s [1-5]. Such problem gained a renewed interest in the context of the transport properties of graphene and related materials [6, 7]. These materials hold the promise of yielding devices with tailored optical properties, among others [8-10].

We briefly review here the formulation of such a problem, as well as the related issue of the calculation of the conductivity of a $2 \mathrm{~d}$ material, so as to apply the underlying results to the problem of radiation losses by fast-particles traversing graphene. We conclude that the signal emitted by graphene is too small to be of use, but such a result may be modified by considering a $2 \mathrm{~d}$ material with a much larger in-plane AC conductivity.

\section{Definition of the screening tensor}

We consider a $2 \mathrm{~d}$ material, e.g. graphene, embedded in a dielectric medium of relative electric and magnetic permittivities, $\varepsilon_{m}$ and $\mu_{m}$. The said medium occupies the $z=0$ plane. The simplest case is that of a suspended material in the vacuum, when $\varepsilon_{m}=\mu_{m}=1$. If a set of external sources generate an applied electric field $\mathbf{E}^{\text {ext }}(\mathbf{r}, t)$, surface densities of charge and

\footnotetext{
*e-mail: jaime.santos@fisica.uminho.pt
} 
current are induced in the material. At the linear response level, the density of current is given by

$$
\boldsymbol{J}_{\mathrm{c}}(\mathbf{q}, \omega)=\sigma_{L}(\mathbf{q}, \omega) \mathbf{E}_{\| L}(z=0, \mathbf{q}, \omega)+\sigma_{T}(\mathbf{q}, \omega) \mathbf{E}_{\| T}(z=0, \mathbf{q}, \omega),
$$

where $\sigma_{L, T}(\mathbf{q}, \omega)$ are the components of the conductivity tensor of the $2 \mathrm{~d}$ material and $\mathbf{E}_{\| L, T}(z=0, \mathbf{q}, \omega)$ are the corresponding components of the local electric field along the plane of the material (respectively parallel and perpendicular to $\mathbf{q}$ ). Here, we employ a mixed real-Fourier space representation, which takes advantage of the translation symmetry in the $x y$ plane.

Such field is related to the induced density of current (1) by (with $v=c / \sqrt{\mu_{m} \varepsilon_{m}}$ )

$$
\mathbf{E}_{\|}(z=0, \mathbf{q}, \omega)=\mathbf{E}_{\|}^{\mathrm{ext}}(z=0, \mathbf{q}, \omega)+\frac{Z_{m}}{2 \sqrt{1-q^{2} v^{2} / \omega^{2}}}\left[\boldsymbol{J}_{\mathrm{c}}(\mathbf{q}, \omega)-\frac{v^{2}\left(\mathbf{q} \cdot \boldsymbol{J}_{\mathrm{c}}(\mathbf{q}, \omega)\right) \mathbf{q}}{\omega^{2}}\right],
$$

where $\omega>q v$ and where the solution of the Maxwell equations was obtained using the Coulomb gauge. Here, $Z_{m}=\sqrt{\frac{\mu_{0} \mu_{m}}{\varepsilon_{0} \varepsilon_{m}}}$ is the impedance of the surrounding medium. As will be explained below, the choice of the gauge is not completely immaterial (for reasons of intelligibility), even if the distribution of the fields is of course independent from it.

Expressing the induced current in terms of the local field using (1), we can obtain a relation between the applied field and the local field. Defining the components of the screening tensor

we obtain the result

$$
\zeta_{L, T}(\mathbf{q}, \omega)=\frac{E_{\| L, T}^{\mathrm{ext}}(z=0, \mathbf{q}, \omega)}{E_{\| L, T}(z=0, \mathbf{q}, \omega)},
$$

$$
\zeta_{L}(\mathbf{q}, \omega)=1+\frac{1}{2} Z_{m} \sigma_{L}(\mathbf{q}, \omega) \sqrt{1-q^{2} v^{2} / \omega^{2}}
$$

and

$$
\zeta_{T}(\mathbf{q}, \omega)=1+\frac{Z_{m} \sigma_{T}(\mathbf{q}, \omega)}{2 \sqrt{1-q^{2} v^{2} / \omega^{2}}}
$$

where retardation effects are included.

If $\omega<q v$, one employs the continuation $\sqrt{1-q^{2} v^{2} / \omega^{2}}=i(v / \omega) \sqrt{q^{2}-\omega^{2} / v^{2}}$ in the above formulas, as determined by the condition of causality. As in the case of a $3 \mathrm{~d}$ medium [11], the knowledge of the conductivity of a $2 \mathrm{~d}$ material is sufficient to determine its screening properties, even if the relations obtained are different in the former and latter cases.

It can be shown that if a plane electromagnetic wave impinges on the $2 \mathrm{~d}$ material, the transmission coefficients for its $\mathrm{p}$ and $\mathrm{s}$ components are related to the components of the screening tensor by $t_{p, s}(\mathbf{q}, \omega)=\zeta_{L, T}^{-1}(\mathbf{q}, \omega)$ (with $q=\frac{\omega}{v} \sin \theta$, where $\theta$ is the angle of incidence of the wave) [12]. Such result can be understood if we interpret the incident wave as the applied field and the transmitted wave (on the other side of the interface) as the local field.

\section{Linear response relations and RPA}

The relations obtained above are of little use if one does not provide an explicit expression for the components of the conductivity tensor of the $2 \mathrm{~d}$ material. One describes it by the Hamiltonian of a many-particle system, in which carriers interact via the Coulomb force, to which one adds a term coupling to an external scalar potential and a term coupling to a vector potential, determined self-consistently [13].

$$
\widehat{H}=\frac{1}{2 m} \sum_{i}\left[\widehat{\mathbf{p}}_{i}-q_{e} \mathbf{A}_{\|}\left(z=0, \widehat{\boldsymbol{\rho}}_{i}, t\right)\right]^{2}+\sum_{i} U\left(\widehat{\boldsymbol{\rho}}_{i}\right)+\frac{1}{2} \sum_{i \neq j} V\left(\widehat{\boldsymbol{\rho}}_{i}-\widehat{\boldsymbol{\rho}}_{j}\right)+q_{e} \sum_{i} \phi_{\mathrm{ext}}\left(z=0, \widehat{\boldsymbol{\rho}}_{i}, t\right),
$$


where $q_{e}$ is the carriers charge, $U(\rho)$ is the potential of the underlying lattice and $V(\rho)=\frac{q_{e}^{2}}{4 \pi \epsilon_{0} \rho}$ is the Coulomb interaction between the charged particles. The potential $\phi_{\text {ext }}(z=0, \rho, t)$ is the applied external potential. At the linear response level in the external scalar potential and the self-consistent vector potential, the induced current and charge densities are given by

$$
\begin{gathered}
J_{c}^{\alpha}(\mathbf{q}, \omega)=q_{e}^{2}\left[\left(\chi^{\alpha \beta}(\mathbf{q}, \omega)-\frac{n_{0}}{m} \delta^{\alpha \beta}\right) A_{\|}^{\beta}(z=0, \mathbf{q}, \omega)-\chi^{\alpha 0}(\mathbf{q}, \omega) \phi_{\mathrm{ext}}(z=0, \mathbf{q}, \omega)\right], \\
\rho_{c}(\mathbf{q}, \omega)=q_{e}^{2}\left[\chi^{0 \beta}(\mathbf{q}, \omega) A_{\|}^{\beta}(z=0, \mathbf{q}, \omega)-\chi(\mathbf{q}, \omega) \phi_{\mathrm{ext}}(z=0, \mathbf{q}, \omega)\right],
\end{gathered}
$$

where $\alpha, \beta$ designate the Cartesian indices (summation over repeated indices is implied) for the components of the current operator and 0 refers to density operator and where these response functions, $\chi^{\alpha \beta}(\mathbf{q}, \omega), \chi^{\alpha 0}(\mathbf{q}, \omega), \chi^{0 \beta}(\mathbf{q}, \omega)$ and $\chi(\mathbf{q}, \omega)$ can be expressed, in the case of an isotropic system, in terms of only two quantities, the density-density response function, $\chi(\mathbf{q}, \omega)$, and the current-current transverse response function, $\chi^{T}(\mathbf{q}, \omega)$.

This approximation scheme, in which the Coulomb interaction is treated exactly, but where the transverse degrees of freedom of the electromagnetic field are treated selfconsistently avoids the explicit inclusion of magnetic interactions (of order $v^{2} / c^{2}$ ) in the Hamiltonian given by equation (6). Furthermore, do note that $A_{\| L}^{\alpha}(z=0, \mathbf{q}, \omega) \neq 0$ in the Coulomb gauge, as $\mathbf{q}$ is merely the projection of the full wave-vector in the $x y$ plane, so we need to keep such a component in equations (7) and (8). This gauge is not only the most adequate to treat the problem in the approximation scheme of Bohm and Pines [13], it also emphasises the distinction between two and three-dimensional materials.

One can show that the conductivities are related to the response functions by

$$
\begin{aligned}
\sigma_{L}(\mathbf{q}, \omega) & =-\frac{i \omega q_{e}^{2}}{q^{2}} \cdot \frac{\chi(\mathbf{q}, \omega)}{1-\frac{q_{e}^{2}}{2 \epsilon_{0} \varepsilon_{m} q} \chi(\mathbf{q}, \omega)}, \\
\sigma_{T}(\mathbf{q}, \omega) & =\frac{q_{e}^{2}}{i \omega}\left(\chi^{T}(\mathbf{q}, \omega)-\frac{n_{0}}{m}\right),
\end{aligned}
$$

where $n_{0}$ is the total electronic density in the material.

If one substitutes these relations in equations (4) and (5), one can obtain an expression for the components of the screening tensor in terms of the response functions as well. The zeros of such components determine the eigen-frequencies of the excitations (plasmons) of the $2 \mathrm{~d}$ material. Static screening may also be considered [14, 15].

These response functions can now be calculated using a given approximation scheme. In the particular case of the Random Phase Approximation (RPA), it is assumed that the system responds to the self-consistent vector and scalar potentials with the response function of the free-carriers. In that case, equations (9) and (10) reduce to

$$
\begin{aligned}
\sigma_{L}(\mathbf{q}, \omega) & =-\frac{i \omega q_{e}^{2}}{q^{2}} \cdot \chi_{0}(\mathbf{q}, \omega) \\
\sigma_{T}(\mathbf{q}, \omega) & =\frac{q_{e}^{2}}{i \omega}\left(\chi_{0}^{T}(\mathbf{q}, \omega)-\frac{n_{0}}{m}\right)
\end{aligned}
$$

where $\chi_{0}(\mathbf{q}, \omega)$ and $\chi_{0}^{T}(\mathbf{q}, \omega)$ are the response functions of the quasi-particles in the material, in the absence of the Coulomb interaction. One can further read from equation (11) that the irreducible susceptibility, given by the second quotient in equation (9), reduces in the RPA to the response function of free carriers, $\chi_{0}(\mathbf{q}, \omega)$. 
An illustrative case is that of neutral graphene, for which one has, in the low wave-vector and low frequency limit [16, 17],

$$
\begin{aligned}
\chi_{0}(\mathbf{q}, \omega) & =\frac{q^{2}}{4 \hbar v_{F} \sqrt{q^{2}-\omega^{2} / v_{F}^{2}}}, \\
\chi_{0}^{T}(\mathbf{q}, \omega) & =\frac{n_{0}}{m}-\frac{v_{F}}{4 \hbar} \sqrt{q^{2}-\omega^{2} / v_{F}^{2}},
\end{aligned}
$$

where $v_{F}$ is the Fermi velocity of the material. The use of equations (11) to (14) in equations (4) and (5) is sufficient to determine the transmission factors of neutral free-standing graphene [12], within the framework of the RPA.

\section{Energy loss through radiation by a fast charged particle traversing graphene}

For a $2 \mathrm{~d}$ material excited by an external field, the energy radiated per solid angle and per frequency interval is given by [18]

$$
\mathcal{S}(\theta, \phi, \omega)=\frac{\mu_{0} \mu_{m} \omega^{2}}{16 \pi^{3} v}\left(\left|\boldsymbol{J}_{\mathrm{c} L}\left(\mathbf{q}_{\rho}, \omega\right)\right|^{2} \cos ^{2} \theta+\left|\boldsymbol{J}_{\mathrm{c} T}\left(\mathbf{q}_{\rho}, \omega\right)\right|^{2}\right)
$$

where the components of the induced current are given by equation (1), $\mathbf{q}_{\rho}=\frac{\omega}{v} \sin \theta \hat{\boldsymbol{\rho}}$, with $\hat{\rho}$ being the polar versor in the plane, and where $\theta$ and $\phi$ are the altitude and azimuthal angles. Expressing the local electric field in the material, as given in equation (1), in terms of the applied field using the components of the screening tensor, introduced in equations (4) and (5), we obtain

$$
\begin{aligned}
\mathcal{S}(\theta, \phi, \omega)= & \frac{\mu_{0} \mu_{m} \omega^{2}}{16 \pi^{3} v}\left(\frac{\left|\sigma_{L}\left(\mathbf{q}_{\rho}, \omega\right)\right|^{2}\left|E_{\| L}^{\operatorname{ext}}\left(z=0, \mathbf{q}_{\rho}, \omega\right)\right|^{2}}{\left|\zeta_{L}\left(\mathbf{q}_{\rho}, \omega\right)\right|^{2}} \cos ^{2} \theta\right. \\
& \left.+\frac{\left|\sigma_{T}\left(\mathbf{q}_{\rho}, \omega\right)\right|^{2}\left|E_{\| T}^{\operatorname{ext}}\left(z=0, \mathbf{q}_{\rho}, \omega\right)\right|^{2}}{\left|\zeta_{T}\left(\mathbf{q}_{\rho}, \omega\right)\right|^{2}}\right) .
\end{aligned}
$$

In order to consider the profile of radiation due to the passage of a fast-travelling charged particle through the $2 \mathrm{~d}$ material, we substitute the known expressions for the components of the (applied) field produced by the particle, assumed in uniform motion [19], in equation (16). In the case of doped graphene, we may further use in such an equation the expressions for the conductivity obtained in an hydrodynamic approximation [20], which corresponds to the low wave-vector and low frequency limit of the linear response theory discussed in section 3

$$
\begin{aligned}
\sigma_{L}(\mathbf{q}, \omega) & =\frac{i \omega \sigma_{0}}{\tau_{F}\left[\omega^{2}-q^{2} v_{F}^{2} / 2+i \omega\left(1 / \tau_{F}+q^{2} v_{F}^{2} \tau_{v} / 4\right)\right]}, \\
\sigma_{T}(\mathbf{q}, \omega) & =\frac{i \sigma_{0}}{\tau_{F}\left[\omega+i\left(1 / \tau_{F}+q^{2} v_{F}^{2} \tau_{v} / 4\right)\right]}
\end{aligned}
$$

where $\tau_{F}$ and $\tau_{v}$ are quasi-particle lifetimes, $\sigma_{0}=\frac{e^{2}\left|\varepsilon_{F}\right| \tau_{F}}{\pi \hbar^{2}}$ is the DC Drude conductivity of graphene, and where $\varepsilon_{F}$ is the Fermi energy of the material (for the case of undoped graphene, the relevant formulas were already given in section 3, within the realm of the RPA). Integrating over frequency, we can obtain the energy emitted per solid angle. A plot of the emission pattern is given in figure 1. Similar results were obtained in [21, 22]. 


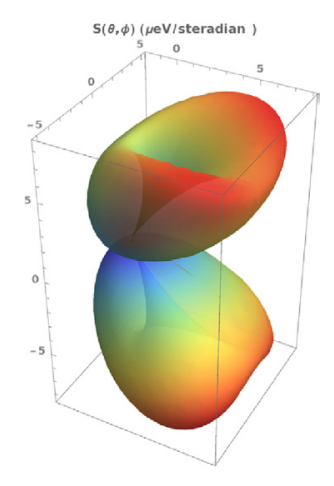

Figure 1. Energy emitted per steradian for an ultra-relativistic electron (with $\beta=0.9$ ) traversing a sheet of mono-layer graphene $\left(\varepsilon_{F}=0.5 \mathrm{eV}\right.$ and $\left.\tau_{v}=\tau_{F}=4.1 \times 10^{-12} \mathrm{~s}\right)$ travelling in the $x z$ plane, at an angle of $30^{\circ}$ from the vertical.

\section{Conclusion}

We considered the pattern of radiation emitted by graphene traversed by a fast charged particle. The low values obtained for the emitted energy require, for the purpose of particle detection, the use of materials possessing high effective AC conductivities, possibly $2 \mathrm{~d}$ semiconductors [23] or thin magnetic films with off-plane magnetisation and a high paramagnetic susceptibility [24].

One should note however, that the emission efficiency of suspended $2 \mathrm{~d}$ passive materials tends to a limiting value for materials with a high conductivity, as follows from equation (16), since the components of the conductivity tensor appear both in the numerator as well as in the denominator of the two terms of this expression, see equations (4) and (5). Moreover, the real part of the screening factors is always larger than one in the region $\omega>q v$, as the real part of the conductivity is always positive in thermodynamic equilibrium, and hence no resonances are to be seen in this region of frequency space.

Acknowledgements: We acknowledge discussions with K. Thygesen, K. Kasbjerg, S. Mikhailov, B. Amorim, P. Cosme, H. Terças, P. Ribeiro and S. Kirchner. J.E.S. thanks support by the European Structural and Investment Funds in the FEDER component, through the Operational Programme for Competitiveness and Internationalization (COMPETE 2020) [under the Project GNESIS - Graphenest's New Engineered System and its Implementation Solutions; Funding Reference: POCI-01-0247-FEDER-033566], European Regional Development Fund, and would also like to thank the hospitality of the Center for Nanostructured Graphene at the Technical University of Denmark, where most of this research was conducted. The CNG is sponsored by the Danish National Research Foundation through Project No. DNRF103. This work was also supported by the Portuguese Foundation for Science and Technology (FCT) in the framework of the Strategic Funding UID/FIS/04650/2019.

\section{References}

[1] R. H. Ritchie, Phys. Rev. 106, 874 (1957)

[2] F. Stern, Phys. Rev. Lett 18, 546 (1967)

[3] K. W. Chiu and J. J. Quinn, Phys. Rev. 9, 4724 (1974)

[4] J. E. Sipe, V. C. Y. So, M. Fukui and G. I. Stegeman, Phys. Rev. 21, 4389 (1980) 
[5] P. Hawrylak, G. Eliasson and J. J. Quinn, Phys. Rev. 37, 10187 (1988)

[6] S. A. Mikhailov and K. Ziegler, Phys. Rev. Lett. 99, 016803, (2007)

[7] V. N. Kotov, B. Uchoa, V. M. Pereira, F. Guinea and A. H. Castro Neto, Rev. Mod. Phys. 84, 1067 (2012)

[8] F. Xia, H. Wang, D. Xiao, M. Dubey and A. Ramasubramaniam, Nature Photonics 8, 899 (2014)

[9] K. S. Novoselov, A. Mishchenko, A. Carvalho and A. H. Castro Neto, Science 353, aac9439 (2016)

[10] S. Xiao, X. Zhu, B.-H. Li and N. A. Mortensen, Front. Phys. 11, 117801 (2016)

[11] D. Pines and P. Noziéres, The theory of quantum liquids, vol. I (Walter Benjamin, New York, 1966) 251

[12] T. Stauber, N. M. R. Peres and A. K. Geim, Phys. Rev. B 78, 085432 (2008)

[13] D. Bohm and D. Pines, Phys. Rev. 82, 625 (1951)

[14] T. Ando, J. Phys. Soc. Jpn., 75, 074716 (2006)

[15] P. Cudazzo, I. V. Tokatly and A. Rubio, Phys. Rev. B 84, 085406 (2011)

[16] K. W.-K. Shung, Phys. Rev. B 34, 979 (1986)

[17] A. Principi, M. Polini, and G. Vignale, Phys. Rev. B 80, 075418 (2009)

[18] J. Schwinger, L. L. DeRaad, K. A. Milton, and W. Tsai, Classical Electrodynamics (Perseus Books, Reading, Massachusetts, 1998) 378

[19] J. D. Jackson, Classical Electrodynamics (John Wiley \& Sons, New York, 1999) 560

[20] A. Principi, G. Vignale, M. Carrega, and M. Polini, Phys Rev B 93, 125410 (2016)

[21] Z. L. Miskovic, S. Segui, J. L. Gervasoni, and N. R. Arista, Phys. Rev. B 94, 125414 (2016)

[22] K. Akbari, S. Segui, Z. L. Miskovic, J. L. Gervasoni, and N. R. Arista, Phys. Rev. B 98, 195410 (2018)

[23] T. Mueller and E. Malic, npj 2D Materials and Applications 2, 29 (2018)

[24] C. Gong, L. Li, Z. Li, H. Ji, A. Stern, Y. Xia, T. Cao, W. Bao, C. Wang, Y. Wang, Z. Q.

Qiu, R. J. Cava, S. G. Louie, J. Xia and X. Zhang, Nature 546, 265 (2017) 